\title{
On Timed Alternating Simulation for Concurrent Timed Games
}

\author{
Laura Bozzelli ${ }^{1}$, Axel Legay ${ }^{1}$, Sophie Pinchinat ${ }^{1}$
}

IRISA, Campus de Beaulieu, 35042 Rennes Cedex, FRANCE.

\begin{abstract}
We address the problem of alternating simulation refinement for concurrent timed games (TG). We show that checking timed alternating simulation between TG is EXPTIME-complete, and provide a logical characterization of this preorder in terms of a meaningful fragment of a new logic, TAMTL*. TAMTL* is an action-based timed extension of standard alternating-time temporal logic ATL*, which allows to quantify on strategies where the designated player is not responsible for blocking time. While for full TAMTL*, model-checking TG is undecidable, we show that for its fragment TAMTL, corresponding to the timed version of ATL, the problem is instead in EXPTIME.
\end{abstract}

\section{Introduction}

Refinement preorders constitute the standard mathematical approach to formalize the relation between abstract and concrete versions of the same system. Intuitively, an implementation $I$ refines an abstraction $A$ when each behavior of $I$ is allowed by $A$. Refinement usually comes together with a logical setting to formally express the requirements preserved by the preorder. The goal is to ensure that the properties proved about the abstract description continue to hold in the refined version (i.e., the implementation). This scenario may arise either because the design is being carried out in an incremental fashion, or because the system is too complex and an abstraction needs to be used to verify its properties.

In the design and analysis of reactive and distributed component-based systems, refinement usually refers to a single component, whose behavior depends on assumptions on its environment (the other components). In this context, traditional refinement preorders, like simulation, are inappropriate because they do not distinguish between the behaviors of the component and those of its environment; so that, refinement also restrict the environment behaviors. Recently, $[5,10,8]$ have addressed this problem and succeeded in an elegant solution for finite-state systems based on the game paradigm: the system is modeled by a multi-player finite-state concurrent game, where at each step, the next state is determined by considering the "intersection" between the choices (behavioral options) made simultaneously and independently by all the players (the components). Thus, one can keep all assumptions about a component separated from those of its environment. In this framework, simulation refinement becomes alternating simulation [5], a preorder which exploits the game setting and is defined according to a designated player (component): ${ }^{*}$ an implementation $I$ refines an abstraction $A$ of the same component whenever any possible behavioral option of $I$ is allowed by $A$, and controvariantly, any possible behavioral option of the environment of $A$ is allowed by the environment of $I$. In this way, the refinement restricts the component behaviors without restricting the permissible environment behaviors.

*or, more in general, w.r.t. any subset of players (coalitions)

(C) Bozzelli, Legay, Pinchinat; licensed under Creative Commons License-NC-ND.

Foundations of Software Technology and Theoretical Computer Science (Kanpur) 2009.

Editors: Ravi Kannan and K. Narayan Kumar; pp 85-96

Leibniz International Proceedings in Informatics (LIPIcs), Schloss Dagstuhl - Leibniz-Zentrum für Informatik, Germany.

Digital Object Identifier: 10.4230/LIPIcs.FSTTCS.2009.2309 
While classical simulation preserves universal fragments of standard branching temporal logics designed for closed systems such as CTL* [11], alternating simulation for a given player preserves expressive fragments of alternating-time temporal logics designed for open systems such as ATL* [5, 4]. The latter is a convenient formalism for component-based systems modeled by finite-state concurrent games, where properties need to be guaranteed by a player irrespective of the behavior of the other players.

Our contribution. We address the problem of refinement for real-time component-based systems, agreeing on the crucial role of timed information in practical applications, e.g. in embedded-system applications. We extend the notion of alternating simulation refinement for finite-state concurrent games to the setting of (perfect-information) timed concurrent games (TG) with the element of surprise introduced in [9]. In this setting, at each step, players choose simultaneously and independently moves consisting of delayed actions: the move with the smallest delay is carried out and determines the next state (if the smallest delay is proposed by several players, then the move of one of them is chosen nondeterministically). Moreover, we propose the new logic TAMTL* as a language for specifying properties of timed component-based systems modeled by TG. TAMTL* is a real-time action-based extension of $\mathrm{ATL}^{*}$, in which the temporal operators correspond to those of the timed linear-time temporal logic MTL [13]. Differently from the known real-time extension of ATL* , namely TATL* [12], which is based on a dense-time continuous semantic (the system is observed at any point in time), we adopt a dense-time pointwise semantics (the system is observed through events) [16]. Furthermore and more importantly, we generalize the class of atomic formulas of MTL by introducing the notion of (timed) multi-action constraint. Intuitively, such constraints express requirements on the "observable" part of single steps along TG runs, i.e., the delay-action chosen by each player and the player which is selected in the current step. In this way we can directly express important properties such as the existence of reasonable strategies, that are strategies where the designated player is not responsible for blocking time progress. In TATL*, this is not directly possible: to express the above requirement we have to artificially extend the infinite labeled transition system (LTS) of the given TG in order to obtain another LTS that cannot be associated to any TG specification. Our main results are the following:

1. We show that checking timed alternating simulation between $T G$ for a given player is EXPTIME-complete. The upper bound is proved by a non-trivial generalization of the region-abstraction approach used for checking timed simulation/bisimulation [7, 17]. The matching lower bound is shown by an easy and linear-time reduction from the problem of checking timed simulation, which is known to be EXPTIME-hard [14].

2. We provide a logical characterization of timed alternating simulation for a given player $\sigma$ in terms of a meaningful fragment, $\sigma$-TAMTL ${ }_{P}^{*}$, of TAMTL*, where strategy quantifiers are parameterized by $\sigma$ and negation applies only to multi-action constraints. We show that a TG $\mathcal{A}$ is timed $\sigma$-simulated by a TG $\mathcal{B}$ precisely when each $\sigma$-TAMTL ${ }_{P}^{*}$ formula that holds in $\mathcal{A}$ also holds in $\mathcal{B}$. To the best of our knowledge, this is the first paper that provides a full logical characterization for a timed refinement preorder.

3. While for unrestricted TAMTL*, model checking TG is undecidable (since TAMTL* subsumes MTL over infinite words[15]), we show that for its fragment TAMTL, where each temporal operator is immediately preceded by a strategy quantifier, the problem 
is instead in EXPTIME. To do so, for each player $\sigma$, we associate to the given TG a region-abstraction finite-state turn-based game $G_{\sigma}$, and recursively reduce the problem to solving the games $G_{\sigma}$ w.r.t. regular objectives. Compared to the TATL model checking algorithm in [12], our approach is direct and provides more insight on TG.

Here, we restrict our attention to the two-player case, but all results can be extended to the multi-player setting, where players play in coalitions. Details of this extension are deferred to the full version of this paper.

Related work. Refinement of real-time closed systems has been addressed in many papers (e.g. [3, 1, 17]), where systems are modeled by standard timed automata (TA) [3]. Timed language containment for TA is undecidable [3], while timed simulation [1, 17] between TA, which preserves the universal fragment of timed CTL (TCTL) [2], is EXPTIME-complete [17, 14]. For the open system setting, we are only aware of the recent work of Bulychev et al. [6], who propose timed simulation preorders for two-player timed games where partial observability is also taken into account. However, the games exploited there are asymmetric, which prevents a natural extension to the multi-player setting. Moreover, there are some significant restrictions on the model. For example, a player is enforced to play a discrete action if the invariant at the current location expires. Furthermore, their notion of preorder differs from ours in at least one crucial point: in their case, there is no interaction between the choices of opponent players in the underlying simulation game.

\section{Preliminaries}

\subsection{Concurrent Timed Games}

Let $\mathbb{R}_{\geq 0}$ be the set of non-negative reals and $\mathbb{Q}_{\geq 0}$ be the set of non-negative rational numbers. Fix a finite set of clock variables $X$. The set $C(X)$ of clock constraints (over $X$ ) is the set of boolean combinations of formulas of the form $x \sim c$, where $x \in X, c$ is a natural number, and $\sim \in\{\leq,<\}$. A (clock) valuation (over $X$ ) is a function $v: X \rightarrow \mathbb{R}_{\geq 0}$ that maps every clock to a non-negative real number. Whether a valuation $v$ satisfies a clock constraint $g \in C(X)$, denoted $v \models g$, is defined in a natural way. For $t \in \mathbb{R}_{\geq 0}$, the valuation $v+t$ is defined as $(v+t)(x)=v(x)+t$ for all $x \in X$. For $Y \subseteq X$, the valuation $v[Y:=0]$ is defined as $(v[Y:=0])(x)=0$ if $x \in Y$ and $(v[Y:=0])(x)=v(x)$ otherwise.

Definition 1.[3] A timed transition table (TT) is a tuple $\mathcal{T}=\langle$ Act, $X, Q, \Delta$, Inv $\rangle$, where Act is a finite set of actions, $Q$ is a finite set of locations, $\Delta \subseteq Q \times($ Act $\cup\{\perp\}) \times C(X) \times 2^{X} \times Q$ is a finite transition relation, where $\perp \notin$ Act is the null action, and Inv $: Q \rightarrow C(X)$ maps each location to an invariant. We require that for each $q \in Q$, there is exactly one transition $\left(q, \perp, g, Y, q^{\prime}\right)$ from $q$ associated with the null action; moreover, $q^{\prime}=q, g=$ true, and $Y=\varnothing$.

A state of $\mathcal{T}$ is a pair $(q, v)$ such that $q \in Q, v$ is a valuation, and the invariant at location $q$ is satisfied by $v$, i.e. $v \models \operatorname{Inv}(q)$. The TT $\mathcal{T}$ induces an infinite-state labeled transition system (LTS) $\llbracket \mathcal{T} \rrbracket=\langle S, \rightarrow\rangle$ over the set of labels $\mathbb{R}_{\geq 0} \times($ Act $\cup\{\perp\}) \times \Delta$, where $S$ is the set of $\mathcal{T}$-states, and the set of labeled edges $\rightarrow \subseteq S \times\left[\mathbb{R}_{\geq 0} \times(\right.$ Act $\left.\cup\{\perp\}) \times \Delta\right] \times S$ is defined as: $(q, v) \stackrel{t, a, \delta}{\longrightarrow}\left(q^{\prime}, v^{\prime}\right)$ iff $\delta=\left(q, a, g, Y, q^{\prime}\right), v+t \models g, v^{\prime}=(v+t)[Y:=0]$, and $v+t^{\prime} \models \operatorname{Inv}(q)$ for each $0<t^{\prime} \leq t$. Note that if $(q, v) \stackrel{t, \perp, \delta}{\longrightarrow}\left(q^{\prime}, v^{\prime}\right)$, then $q^{\prime}=q$ and $v^{\prime}=v+t$. 
DEFINITION 2.[9] $A$ (two-player concurrent) timed game (TG) is a tuple $\mathcal{A}=\left\langle\mathcal{T}, s^{0}\right.$, Act $_{0}$, $\left.A c t_{1}\right\rangle$, where $\mathcal{T}=\langle A c t, X, Q, \Delta$, Inv $\rangle$ is a TT, $s^{0}$ is a designated initial state of $\mathcal{T}$ whose clock values are in $\mathbb{Q}_{\geq 0}$, and $\left\{A c t_{0}, A c t_{1}\right\}$ is a partition of Act with $A c t_{0}, A c t_{1} \neq \varnothing$.

A state of $\mathcal{A}$ is a state of $\llbracket \mathcal{T} \rrbracket$. For each $\sigma \in\{0,1\}$, let $\operatorname{Act}_{\sigma}^{\perp}=\operatorname{Act}_{\sigma} \cup\{\perp\}$. Intuitively, $A c t_{\sigma}^{\perp}$ represents the set of actions for player $\sigma$. The set of moves $\operatorname{Mov}_{\mathcal{A}}(\sigma)$ of player $\sigma$ is given by $\mathbb{R}_{\geq 0} \times A c t t_{\sigma}^{\perp} \times \Delta$. For a state $s$, the set of moves available to player $\sigma$ in $s$, written $\operatorname{Mov}_{\mathcal{A}}(\sigma, s)$, is the set of moves $(t, a, \delta) \in \operatorname{Mov}_{\mathcal{A}}(\sigma)$ such that $s \stackrel{t, a, \delta}{\longrightarrow} s^{\prime}$ for some state $s^{\prime}$, which is uniquely determined and is denoted by $\operatorname{Next}_{\mathcal{A}}(s,\langle t, a, \delta\rangle)$. Observe that $\operatorname{Mov}_{\mathcal{A}}(\sigma, s)$ is not empty since $(0, \perp,(q, \perp$, true $, \varnothing, q)) \in \operatorname{Mov}_{\mathcal{A}}(\sigma, s)$, where $q$ is the location of $s$.

The timed game is intuitively played as follows. In each state $s$, each player $\sigma$ chooses a move $(t, a, \delta) \in \operatorname{Mov}_{\mathcal{A}}(\sigma, s)$ indicating that the player wants to play the transition $\delta$ associated with the action $a$ after a delay of $t$ time units. The null action $\perp$ signifies the player's intention to remain idle for the specified time delay. The move with the shorter proposed time delay determines the next state of the game; if both player propose the same delay, then one of the chosen moves occurs non-deterministically. An outcome of the game corresponds to an infinite path of $\llbracket \mathcal{T} \rrbracket$ augmented with additional information. Before formalizing these notions, we recall that in the standard definition of TG (see e.g. [9]) a move of a player just consists of a timed delay followed by an action. This because the underlying TT is assumed to be time-deterministic, i.e. for each $(t, a) \in \mathbb{R}_{\geq 0} \times$ Act and state $s$, there is at most one transition $\delta$ such that $s \stackrel{t, a, \delta}{\longrightarrow} s^{\prime}$. Here, we have removed this restriction. Thus, to uniquely determine the next state, a player has to specify also the transition to be taken.

For moves $\left(t_{0}, a_{0}, \delta_{0}\right) \in \operatorname{Mov}_{\mathcal{A}}(0, s)$ and $\left(t_{1}, a_{1}, \delta_{1}\right) \in \operatorname{Mov}_{\mathcal{A}}(1, s)$, the joint destination move, written $\operatorname{JDM}\left(\left\langle t_{0}, a_{0}, \delta_{0}\right\rangle,\left\langle t_{1}, a_{1}, \delta_{1}\right\rangle\right)$, is $\left\{\left\langle t_{0}, a_{0}, \delta_{0}\right\rangle,\left\langle t_{1}, a_{1}, \delta_{1}\right\rangle\right\}$ if $t_{0}=t_{1}$, and the singleton $\left\{\left\langle t_{k}, a_{k}, \delta_{k}\right\rangle\right\}$ for the unique $k \in\{0,1\}$ such that $t_{k}<t_{1-k}$ otherwise.

A run of $\mathcal{A}$ is a finite or infinite sequence $\pi=s_{0},\left\langle m_{1}^{0}, m_{1}^{1}, \sigma_{1}\right\rangle, s_{1},\left\langle m_{2}^{0}, m_{2}^{1}, \sigma_{2}\right\rangle, s_{2}, \ldots$ such that for any $k, s_{k} \in S, m_{k+1}^{0} \in \operatorname{Mov}_{\mathcal{A}}\left(0, s_{k}\right), m_{k+1}^{1} \in \operatorname{Mov}_{\mathcal{A}}\left(1, s_{k}\right), \sigma_{k+1} \in\{0,1\}, m_{k+1}^{\sigma_{k+1}} \in$ $\operatorname{JDM}\left(m_{k+1}^{0}, m_{k+1}^{1}\right)$, and $s_{k+1}=\operatorname{Next}_{\mathcal{A}}\left(s_{k}, m_{k+1}^{\sigma_{k+1}}\right)$. For each $k$, we denote by $\pi^{k}$ the suffix-run of $\pi$ starting from state $s_{k}$, and by $\pi[0, k]$ the prefix-run of $\pi$ leading to state $s_{k}$. The duration $\operatorname{DUR}(\pi)$ of $\pi$ is the sum of timestamps of the selected moves $m_{k+1}^{\sigma_{k+1}}$ along $\pi$. An infinite run $\pi$ is divergent if $\operatorname{DUR}(\pi)=+\infty$. Let FRuns be the set of finite runs of $\mathcal{A}$. For $\pi \in$ FRuns, we denote by last $(\pi)$ the last state of $\pi$. A strategy $f_{\sigma}$ for player $\sigma \in\{0,1\}$ is a mapping $f_{\sigma}:$ FRuns $\rightarrow \operatorname{Mov}_{\mathcal{A}}(\sigma)$ assigning to each finite run $\pi$ a move to be proposed by player $\sigma$ at last $(\pi)$ such that $f_{\sigma}(\pi) \in \operatorname{Mov}_{A}(\sigma$, last $(\pi))$. For each state $s$, the set of outcomes of strategy $f_{\sigma}$ from $s$, Outcomes $\mathcal{A}_{\mathcal{A}}\left(\sigma, s, f_{\sigma}\right)$, is the set of all infinite runs $s_{0},\left\langle m_{1}^{0}, m_{1}^{1}, \sigma_{1}\right\rangle, s_{1},\left\langle m_{2}^{0}, m_{2}^{1}, \sigma_{2}\right\rangle, s_{2} \ldots$ such that $s_{0}=s$, and for each $k \geq 0, f_{\sigma}\left(s_{0},\left\langle m_{1}^{0}, m_{1}^{1}, \sigma_{1}\right\rangle, s_{1}, \ldots s_{k}\right)=m_{k+1}^{\sigma}$. Let $\pi=$ $s_{0},\left\langle m_{1}^{0}, m_{1}^{1}, \sigma_{1}\right\rangle, s_{1},\left\langle m_{2}^{0}, m_{2}^{1}, \sigma_{2}\right\rangle, s_{2} \ldots$ with $m_{k}^{j}=\left(t_{k}^{j}, a_{k}^{j}, \delta_{k}^{j}\right)$ (for each $j=0,1$ and $k \geq 1$ ). The trace of $\pi$, written trace $(\pi)$, is $\left\langle\left(t_{1}^{0}, a_{1}^{0}\right),\left(t_{1}^{1}, a_{1}^{1}\right), \sigma_{1}\right\rangle,\left\langle\left(t_{2}^{0}, a_{2}^{0}\right),\left(t_{2}^{1}, a_{2}^{1}\right), \sigma_{2}\right\rangle, \ldots$

We are also interested in strategies $f_{\sigma}$ of player $\sigma \in\{0,1\}$ such that player $\sigma$ is not responsible for blocking time progress [9]. Let Blameless ${ }_{\sigma}$ be the set of infinite runs $\pi=$ $s_{0},\left\langle m_{1}^{0}, m_{1}^{1}, \sigma_{1}\right\rangle, s_{1}, \ldots$ such that player $\sigma$ is responsible only for finitely many steps, i.e. such that there is $k \geq 1$ so that for all $j \geq k, \sigma_{j}=1-\sigma$. Note that Blameless $_{\sigma}$ does not distinguish between runs which have the same trace. A strategy $f_{\sigma}$ for player $\sigma$ is reasonable in a state $s$ iff for all runs $\pi$ in Outcomes $_{\mathcal{A}}\left(\sigma, s, f_{\sigma}\right)$, either $\pi$ is divergent or $\pi \in$ Blameless $_{\sigma}$. 


\subsection{The logic TAMTL*}

In this subsection, we introduce a real-time action-based extension of the alternating-time temporal logic ATL* [4], called TAMTL* , based on a dense-time pointwise semantics.

Fix two nonempty and disjoint sets of actions $A c t_{0}$ and $A c t_{1}$. A (timed) multi-action over $\left(\right.$ Act $\left._{0}, A c t_{1}\right)$ is a triple $\theta=\left\langle\left(t_{0}, a_{0}\right),\left(t_{1}, a_{1}\right), \sigma\right\rangle$, where $\left(t_{i}, a_{i}\right) \in \mathbb{R}_{\geq 0} \times A c t_{i}^{\perp}$ for $i=0,1$, $\sigma \in\{0,1\}$, and $t_{\sigma} \leq t_{0}, t_{1}$. Note that the traces of runs in TG on $\left(A c t_{0}, A c t_{1}\right)$ are sequence of multi-actions. A (timed) multi-action constraint $\chi$ is a triple $\chi=\left\langle\left(a_{0}^{\prime}, \sim_{0} c_{0}\right),\left(a_{1}^{\prime}, \sim_{1} c_{1}\right), \sigma^{\prime}\right\rangle$, where $\sigma^{\prime} \in\{0,1\}$ and for $i=0,1, a_{i}^{\prime} \in A c t_{i}^{\perp}, \sim_{i} \in\{=,<, \leq,>, \geq\}$, and $c_{i} \in \mathbb{Q}_{\geq 0}$. The above multi-action $\theta$ satisfies $\chi$, written $\theta \models \chi$, iff $\sigma=\sigma^{\prime}$ and for $i=0,1, a_{i}=a_{i}^{\prime}$ and $t_{i} \sim_{i} c_{i}$.

The sets of state formulas $\varphi$ and path formulas $\psi$ of TAMTL* over $\left(A c t_{0}, A c t_{1}\right)$ are defined as:

$$
\begin{aligned}
& \varphi:=\text { true }|\neg \varphi| \varphi \wedge \varphi|\varphi \vee \varphi|\langle\langle\sigma\rangle\rangle \psi \mid\langle\langle\sigma\rangle\rangle_{r e} \psi \\
& \psi:=\chi|\varphi| \neg \psi|\psi \wedge \psi| \psi \vee \psi\left|\psi \mathcal{U}_{I} \psi\right| \psi \widetilde{\mathcal{U}}_{I} \psi
\end{aligned}
$$

where $\sigma \in\{0,1\},\langle\langle\sigma\rangle\rangle$ and $\langle\langle\sigma\rangle\rangle_{\text {re }}$ are strategy quantifiers, where, intuitively, $\langle\langle\sigma\rangle\rangle_{\text {re }}$ is restricted to $\sigma$-reasonable strategies, $\chi$ is a multi-action constraint, $\mathcal{U}_{I}$ is the constrained strict until operator, where $I$ is an interval with bounds in $\mathbb{Q}_{\geq 0} \cup\{+\infty\}$, and $\widetilde{\mathcal{U}}_{I}$ is the dual of $\mathcal{U}_{I}$. The set of state formulas $\varphi$ forms the language TAMTL*. TAMTL* is interpreted over states of TG. Let $\mathcal{A}$ be a TG over $\left(A c t_{0}, A c t_{1}\right)$, s be a state of $\mathcal{A}$, and $\pi$ be an infinite run of $\mathcal{A}$. For a state formula $\varphi$ and a path formula $\psi$, the satisfaction relations $(\mathcal{A}, s) \models \varphi$ and $(\mathcal{A}, \pi) \models \psi$ are defined by induction as follows (we omit the rules for boolean connectives):

$$
\begin{aligned}
& (\mathcal{A}, s) \models\langle\langle\sigma\rangle\rangle \psi \quad \text { iff there is a strategy } f \text { of player } \sigma \text { such that } \\
& \text { for all } \pi \in \text { Outcomes }_{\mathcal{A}}(\sigma, s, f),(\mathcal{A}, \pi) \models \psi \\
& (\mathcal{A}, s) \models\langle\langle\sigma\rangle\rangle_{\text {re }} \psi \quad \text { iff } \quad \text { there is a reasonable strategy } f \text { of player } \sigma \text { such that for all } \\
& \pi \in \text { Outcomes }_{\mathcal{A}}(\sigma, s, f),(\mathcal{A}, \pi) \models \psi \text { if } \pi \text { is divergent } \\
& (\mathcal{A}, \pi) \models \chi \quad \text { iff } \operatorname{trace}(\pi)=\theta_{0}, \theta_{1}, \ldots \text { and } \theta_{0} \models \chi \\
& (\mathcal{A}, \pi) \models \varphi \quad \text { iff } \pi=s_{0}, \ldots \text { and }\left(\mathcal{A}, s_{0}\right) \models \varphi \\
& (\mathcal{A}, \pi) \models \psi_{1} \mathcal{U}_{I} \psi_{2} \quad \text { iff there is } i>0 \text { such that } \operatorname{DUR}(\pi[0, i]) \in I \text {, } \\
& \left(\mathcal{A}, \pi^{i}\right) \models \psi_{2} \text {, and }\left(\mathcal{A}, \pi^{k}\right) \models \psi_{1} \text { for all } 0<k<i \\
& (\mathcal{A}, \pi) \models \psi_{1} \tilde{\mathcal{U}}_{I} \psi_{2} \quad \text { iff } \quad(\mathcal{A}, \pi) \models \neg\left(\left(\neg \psi_{1}\right) \mathcal{U}_{I}\left(\neg \psi_{2}\right)\right)
\end{aligned}
$$

We write $\mathcal{A} \models \varphi$ to mean that $\left(\mathcal{A}, s^{0}\right) \models \varphi$ for the initial state $s^{0}$ of $\mathcal{A}$. We use some standard shortcuts: $\diamond_{I} \psi:=$ true $\mathcal{U}_{I} \psi$ (eventually), $\square_{I} \psi:=\neg \diamond_{I} \neg \psi$ (always), and $\bigcirc_{I} \psi:=(\neg$ true $) \mathcal{U}_{I} \psi$ (next). We omit the subscript $I$ when $I=\mathbb{R}_{\geq 0}$. We denote by TAMTL the fragment of TAMTL* consisting of the formulas in which every temporal operator is immediately preceded by a strategy quantifier. Moreover, for $\sigma \in\{0,1\}$, let $\sigma$-TAMTL ${ }_{P}^{*}$ be the fragment of TAMTL* (not closed under negation) in which all strategy quantifiers are parameterized by $\sigma$, and negation is applied only to multi-action constraints. Intuitively, $\sigma$-TAMTL ${ }_{P}^{*}$ formulas describe behaviors that player $\sigma$ can enforce no matter what player $1-\sigma$ does. Note that since TG are not determined [9], the dual $\sigma$ - $\widehat{\mathrm{TAMTL}_{P}^{*}}$ of $\sigma$ - $\mathrm{TAMTL}_{P}^{*}$ does not correspond to $(1-\sigma)-$ TAMTL $_{P}^{*}$. By the equivalence below, it follows that in fact $\langle\langle\sigma\rangle\rangle_{r e}$ is a derivate operator in TAMTL* and also in $\sigma$-TAMTL ${ }_{P}^{*}$. However, this does not hold for the logic TAMTL.

$$
\begin{aligned}
& \langle\langle\sigma\rangle\rangle_{\text {re }} \psi \equiv\langle\langle\sigma\rangle\rangle\left(( ( \square \diamond _ { [ 1 , \infty [ } \text { true } ) \rightarrow \psi ) \wedge \left(\neg \left(\square \diamond_{\left.\left[1, \infty[\text { true }) \rightarrow \psi_{\text {blameless }_{\sigma}}\right)\right)}\right.\right.\right. \\
& \psi_{\text {blameless }_{\sigma}}:=\diamond \square\left(\bigvee_{a_{0} \in \text { Act }_{0}^{\perp}} \bigvee_{a_{1} \in \text { Act }_{\perp}^{\perp}}\left\langle\left(a_{0}, \geq 0\right),\left(a_{1}, \geq 0\right), 1-\sigma\right\rangle\right)
\end{aligned}
$$


ExAMPLE 3. Let $A c t_{0}=\{a\}$ and $A c t_{1}=\{b\}$. The 0-TAMTL formula $\langle\langle 0\rangle\rangle_{r e} \square(\langle(a, \geq 0)$, $\left.\left.(b, \geq 0), 0\rangle \rightarrow \nabla_{[1,1]}\langle(a, \geq 0),(b, \geq 0), 1)\right\rangle\right)$ requires that player 0 has a reasonable strategy ensuring that along every its divergent outcome, every $a$-event (i.e., the action $a$ is selected in the current step) is followed one time unit later by a $b$-event.

\section{Timed Alternating Simulation}

In this section, we introduce the notion of timed alternating simulation between TG which generalizes alternating simulation between finite-state concurrent games [5].

Fix two comparable TG $\mathcal{A}=\left\langle\mathcal{T}_{\mathcal{A}}, s_{0}^{\mathcal{A}}, A c t_{0}^{\mathcal{A}}, A c t_{1}^{\mathcal{A}}\right\rangle$ and $\mathcal{B}=\left\langle\mathcal{T}_{\mathcal{B}}, s_{0}^{\mathcal{B}}, A c t_{0}^{\mathcal{B}}, A c t_{1}^{\mathcal{B}}\right\rangle$, i.e. such that $A c t_{0}^{\mathcal{A}}=A c t_{0}^{\mathcal{B}}$ and $A c t_{1}^{\mathcal{A}}=A c t_{1}^{\mathcal{B}}$. Let $S_{\mathcal{A}}$ (resp., $\left.S_{\mathcal{B}}\right)$ be the set of states of $\mathcal{A}($ resp., $\mathcal{B})$.

Definition 4. For a player $\sigma \in\{0,1\}$, a relation $H \subseteq S_{\mathcal{A}} \times S_{\mathcal{B}}$ is a timed alternating simulation for player $\sigma$ from $\mathcal{A}$ to $\mathcal{B}$ iff for all $\left(s_{\mathcal{A}}, s_{\mathcal{B}}\right) \in H$, the following holds:

- for every move $m_{\sigma}^{\mathcal{A}}=\left(t, a, \delta_{\mathcal{A}}\right) \in \operatorname{Mov}_{\mathcal{A}}\left(\sigma, s_{\mathcal{A}}\right)$, there is a matching move $m_{\sigma}^{\mathcal{B}}=\left(t, a, \delta_{\mathcal{B}}\right) \in$ $\operatorname{Mov}_{\mathcal{B}}\left(\sigma, s_{\mathcal{B}}\right)$ such that for every move $m_{1-\sigma}^{\mathcal{B}}=\left(t^{\prime}, b, \delta_{\mathcal{B}}^{\prime}\right) \in \operatorname{Mov}_{\mathcal{B}}\left(1-\sigma, s_{\mathcal{B}}\right)$, there is a matching move $m_{1-\sigma}^{\mathcal{A}}=\left(t^{\prime}, b, \delta_{\mathcal{A}}^{\prime}\right) \in \operatorname{Mov}_{\mathcal{A}}\left(1-\sigma, s_{\mathcal{A}}\right)$ so that for all $i=0,1$,

$$
m_{i}^{\mathcal{A}} \in J D M\left(m_{0}^{\mathcal{A}}, m_{1}^{\mathcal{A}}\right) \text { implies }\left(\operatorname{Next}_{\mathcal{A}}\left(s_{\mathcal{A}}, m_{i}^{\mathcal{A}}\right), \operatorname{Next}_{\mathcal{B}}\left(s_{\mathcal{B}}, m_{i}^{\mathcal{B}}\right)\right) \in H
$$

Note that $m_{i}^{\mathcal{B}} \in J D M\left(m_{0}^{\mathcal{B}}, m_{1}^{\mathcal{B}}\right)$. If there is a timed alternating simulation $H$ for player $\sigma$ from $\mathcal{A}$ to $\mathcal{B}$ such that $\left(s_{0}^{\mathcal{A}}, s_{0}^{\mathcal{B}}\right) \in H$, we say that $\mathcal{B}$ timed $\sigma$-simulates $\mathcal{A}$, and we write $\mathcal{A} \preceq{ }_{\sigma} \mathcal{B}$. Note that $\preceq_{\sigma}$ is a preorder on TG. We can give a game-theoretic interpretation of timed alternating simulation for a player $\sigma \in\{0,1\}$. Consider the following two-player turn-based game whose set of main positions is $S_{\mathcal{A}} \times S_{\mathcal{B}}$. The initial position is $\left(s_{0}^{\mathcal{A}}, s_{0}^{\mathcal{B}}\right)$. Each round consists of five steps as follows. Assume that the current main position is $\left(s_{\mathcal{A}}, s_{\mathcal{B}}\right)$. Then:

1. The antagonist chooses a move $m_{\sigma}^{\mathcal{A}}=\left(t, a, \delta_{\mathcal{A}}\right) \in \operatorname{Mov}_{\mathcal{A}}\left(\sigma, s_{\mathcal{A}}\right)$ of player $\sigma$ in $\mathcal{A}$ available at state $s_{\mathcal{A}}$, and moves to position $p_{1}=\left(s_{\mathcal{A}}, s_{\mathcal{B}}, m_{\sigma}^{\mathcal{A}}\right)$.

2. The protagonist, from $p_{1}$, chooses a matching move $m_{\sigma}^{\mathcal{B}}=\left(t, a, \delta_{\mathcal{B}}\right) \in \operatorname{Mov}_{\mathcal{B}}\left(\sigma, s_{\mathcal{B}}\right)$ of player $\sigma$ in $\mathcal{B}$ available at state $s_{\mathcal{B}}$, and moves to position $p_{2}=\left(s_{\mathcal{A}}, s_{\mathcal{B}}, m_{\sigma}^{\mathcal{A}}, m_{\sigma}^{\mathcal{B}}\right)$.

3. The antagonist, from $p_{2}$, chooses a move $m_{1-\sigma}^{\mathcal{B}}=\left(t^{\prime}, b, \delta_{\mathcal{B}}^{\prime}\right) \in \operatorname{Mov}_{\mathcal{B}}\left(1-\sigma, s_{\mathcal{B}}\right)$ of player $1-\sigma$ in $\mathcal{B}$ available at state $s_{\mathcal{B}}$, and moves to position $p_{3}=\left(s_{\mathcal{A}}, s_{\mathcal{B}}, m_{\sigma}^{\mathcal{A}}, m_{\sigma}^{\mathcal{B}}, m_{1-\sigma}^{\mathcal{B}}\right)$.

4. The protagonist, from $p_{3}$, chooses a matching move $m_{1-\sigma}^{\mathcal{A}}=\left(t^{\prime}, b, \delta_{\mathcal{A}}^{\prime}\right) \in \operatorname{Mov}_{\mathcal{A}}\left(\sigma_{,} s_{\mathcal{A}}\right)$ of player $1-\sigma$ in $\mathcal{A}$ available at state $s_{\mathcal{A}}$, and moves to $p_{4}=\left(s_{\mathcal{A}}, s_{\mathcal{B}}, m_{\sigma}^{\mathcal{A}}, m_{\sigma}^{\mathcal{B}}, m_{1-\sigma}^{\mathcal{B}}, m_{1-\sigma}^{\mathcal{A}}\right)$.

5. The antagonist, from position $p_{4}$, chooses $i=0,1$ such that $m_{i}^{\mathcal{A}} \in J D M\left(m_{0}^{\mathcal{A}}, m_{1}^{\mathcal{A}}\right)$, and moves to the main position $\left(\operatorname{Next}_{\mathcal{A}}\left(s_{\mathcal{A}}, m_{i}^{\mathcal{A}}\right), \operatorname{Next}_{\mathcal{B}}\left(s_{\mathcal{B}}, m_{i}^{\mathcal{B}}\right)\right)$.

If the game proceeds ad infinitum, then the protagonist wins. Otherwise, the game reaches a position from which the protagonist cannot choose in steps 2 or 4 above a matching move, and the antagonist wins. It easily follows that $\mathcal{B}$ timed $\sigma$-simulates $\mathcal{A}$ iff the protagonist has a winning strategy. Note that for each $\sigma \in\{0,1\}$, we have a different turn-based game.

Intuitively, $\mathcal{B}$ timed $\sigma$-simulates $\mathcal{A}$ iff player $\sigma$ is more powerful in game $\mathcal{B}$ than in game $\mathcal{A}$, i.e. each behavior that player $\sigma$ can induce in $\mathcal{A}$, it can also induce in $\mathcal{B}$. The following lemma formalizes this intuition. Let $H \subseteq S_{\mathcal{A}} \times S_{\mathcal{B}}$. For a run $\pi$ of $\mathcal{A}$ and a run $\pi^{\prime}$ of $\mathcal{B}$ having the same length, we write $H\left(\pi, \pi^{\prime}\right)$ to mean that for each prefix-run $\pi[0, k]$ of $\pi$, $\left(\operatorname{last}(\pi[0, k]), \operatorname{last}\left(\pi^{\prime}[0, k]\right)\right) \in H$. 
LEMMA 5. Let $H$ be a timed alternating simulation for player $\sigma \in\{0,1\}$ from $\mathcal{A}$ to $\mathcal{B}$. Then, for all $\left(s_{\mathcal{A}}, s_{\mathcal{B}}\right) \in H$ and strategy $f_{\mathcal{A}}$ of player $\sigma$ in $\mathcal{A}$, there exists a strategy $f_{\mathcal{B}}$ of player $\sigma$ in $\mathcal{B}$ such that for every run $\pi_{\mathcal{B}} \in \operatorname{Outcomes}_{\mathcal{B}}\left(\sigma, s_{\mathcal{B}}, f_{\mathcal{B}}\right)$, there exists a run $\pi_{\mathcal{A}} \in$ Outcomes $\mathcal{A}_{\mathcal{A}}\left(\sigma, s_{\mathcal{A}}, f_{\mathcal{A}}\right)$ so that $H\left(\pi_{\mathcal{A}}, \pi_{\mathcal{B}}\right)$ and $\operatorname{trace}\left(\pi_{\mathcal{A}}\right)=\operatorname{trace}\left(\pi_{\mathcal{B}}\right)$.

EXAMPLE 6. The figure depicts two simple TG $\mathcal{A}$ and $\mathcal{B}$ with $A c t_{0}=\{a\}$ and $A c t_{1}=\{b\}$. Let $s_{\mathcal{A}}^{0}=\left(q_{\mathcal{A}}, v\right)$ and $s_{\mathcal{B}}^{0}=\left(q_{\mathcal{B}}, v\right)$ be the initial states of $\mathcal{A}$ and $B$, where $v$ is any valuation with $v(x) \leq 1$. It easily follows that $\mathcal{B}$ timed 0 -simulates $\mathcal{A}$ and $\mathcal{A}$ timed 1 -simulates $B$, but the vice versa of each of two conditions does not hold. Moreover, note that there exists no (standard) timed simulation from $\mathcal{A}$ to $\mathcal{B}$ and vice versa (w.r.t. the given initial states).

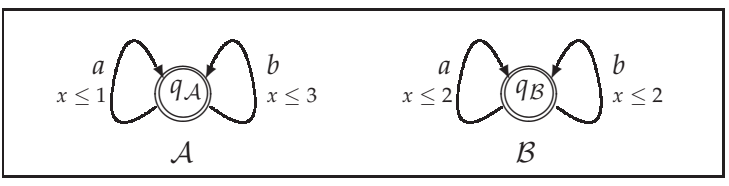

\subsection{Checking Timed Alternating Simulation}

In this subsection, we show that for given comparable TG $\mathcal{A}$ and $\mathcal{B}$, and player $\sigma \in\{0,1\}$, checking whether $\mathcal{A} \preceq_{\sigma} \mathcal{B}$ is decidable via a suitable region abstraction, and the check can be done in exponential time. Fix two comparable TG $\mathcal{A}=\left\langle\mathcal{T}_{\mathcal{A}}, s_{0}^{\mathcal{A}}, A c t_{0}, A c t_{1}\right\rangle$ and $\mathcal{B}=$ $\left\langle\mathcal{T}_{\mathcal{B}}, s_{0}^{\mathcal{B}}, A c t_{0}, A c t_{1}\right\rangle$. Let $S_{\mathcal{A}}$ (resp., $S_{\mathcal{B}}$ ) be the set of states of $\mathcal{A}$ (resp., $\mathcal{B}$ ), and let $X_{\mathcal{A}}$ (resp., $X_{\mathcal{B}}$ ) be the set of clocks of $\mathcal{A}$ (resp., $\mathcal{B}$ ). W.l.o.g. we can assume that $X_{\mathcal{A}} \cap X_{\mathcal{B}}=\varnothing$.

Region equivalence [3]: we denote by $K_{\max }$ the largest constant occurring in the clock constraints of $\mathcal{A}$ and $\mathcal{B}$. Given a clock valuation $v_{\mathcal{A}}$ over $X_{\mathcal{A}}$ and a clock valuation $v_{\mathcal{B}}$ over $X_{\mathcal{B}}$, the clock valuation $v_{\mathcal{A}} \| v_{\mathcal{B}}$ over $X_{\mathcal{A}} \cup X_{\mathcal{B}}$ is defined in the obvious way (recall that $\left.X_{\mathcal{A}} \cap X_{\mathcal{B}}=\varnothing\right)$. For $t \in \mathbb{R}_{\geq 0},\lfloor t\rfloor$ denotes the integral part of $t$ and fract $(t)$ denotes its fractional part. The region equivalence relation over $S_{\mathcal{A}} \times S_{\mathcal{B}}$, written $\approx_{\mathcal{A} \| \mathcal{B}}$, is defined as follows: $\left(\left(q_{\mathcal{A}}, v_{\mathcal{A}}\right),\left(q_{\mathcal{B}}, v_{\mathcal{B}}\right)\right) \approx_{\mathcal{A} \| \mathcal{B}}\left(\left(q_{\mathcal{A}}^{\prime}, v_{\mathcal{A}}^{\prime}\right),\left(q_{\mathcal{B}}^{\prime}, v_{\mathcal{B}}^{\prime}\right)\right)$ iff $q_{\mathcal{A}}=q_{\mathcal{A}}^{\prime}, q_{\mathcal{B}}=q_{\mathcal{B}}^{\prime}$, and for each $x \in X_{\mathcal{A}} \cup X_{\mathcal{B}}$, either both $\left(v_{\mathcal{A}} \| v_{\mathcal{B}}\right)(x),\left(v_{\mathcal{A}}^{\prime} \| v_{\mathcal{B}}^{\prime}\right)(x)>K_{\text {max }}$, or the following holds:

- $\left\lfloor\left(v_{\mathcal{A}} \| v_{\mathcal{B}}\right)(x)\right\rfloor=\left\lfloor\left(v_{\mathcal{A}}^{\prime} \| v_{\mathcal{B}}^{\prime}\right)(x)\right\rfloor$ and $\operatorname{fract}\left(\left(v_{\mathcal{A}} \| v_{\mathcal{B}}\right)(x)\right)=0$ iff $\operatorname{fract}\left(\left(v_{\mathcal{A}}^{\prime} \| v_{\mathcal{B}}^{\prime}\right)(x)\right)=0$;

- for each $y \in X_{\mathcal{A}} \cup X_{\mathcal{B}}$ s.t. $\left(v_{\mathcal{A}} \| v_{\mathcal{B}}\right)(y) \leq K_{\text {max }}, \operatorname{fract}\left(\left(v_{\mathcal{A}} \| v_{\mathcal{B}}\right)(x)\right) \leq \operatorname{fract}\left(\left(v_{\mathcal{A}} \| v_{\mathcal{B}}\right)(y)\right)$ iff $\operatorname{fract}\left(\left(v_{\mathcal{A}}^{\prime} \| v_{\mathcal{B}}^{\prime}\right)(x)\right) \leq \operatorname{fract}\left(\left(v_{\mathcal{A}}^{\prime} \| v_{\mathcal{B}}^{\prime}\right)(y)\right)$ (ordering of the fractional parts).

Let $\operatorname{Reg}_{\mathcal{A} \| \mathcal{B}}$ be the set of equivalence classes of $\approx_{\mathcal{A} \| \mathcal{B}}$, called regions. By [3], $\operatorname{Reg}_{\mathcal{A} \| \mathcal{B}}$ is finite and its size is singly exponential in the sizes of $\mathcal{A}$ and $\mathcal{B}$.

Finite Sampling of $\mathbb{R}_{\geq 0}$ : let $\left(s_{\mathcal{A}}, s_{B}\right) \in S_{\mathcal{A}} \times S_{\mathcal{B}}$ and $x_{1}, \ldots, x_{n}$ be the clocks in $X_{\mathcal{A}} \cup X_{\mathcal{B}}$ whose values $t_{1}, \ldots, t_{n}$ in $\left(s_{\mathcal{A}}, s_{\mathcal{B}}\right)$ are not greater than $K_{\max }$. Assume w.l.o.g. that $\tau_{1} \leq \tau_{2} \leq$ $\ldots \leq \tau_{n}$, where $\tau_{i}=\operatorname{fract}\left(t_{i}\right)$ for $1 \leq i \leq n$. Let $\tau_{0}=0, \tau_{n+1}=1$, and $\min \left(s_{\mathcal{A}}, s_{\mathcal{B}}\right)=$ $\min \left\{\left\lfloor t_{1}\right\rfloor, \ldots,\left\lfloor t_{n}\right\rfloor\right\}$. We consider the following finite set of real numbers:

$\operatorname{Times}\left(s_{\mathcal{A}}, s_{B}\right)=\left\{h-\frac{1}{2}\left(\tau_{i}+\tau_{i+1}\right) \mid i=0, \ldots, n\right.$ and $\left.h=1, \ldots, K_{\max }-\min \left(s_{\mathcal{A}}, s_{\mathcal{B}}\right)\right\} \cup$ $\left\{h-\tau_{i} \mid i=1, \ldots, n\right.$ and $\left.\left.h=1, \ldots, K_{\max }-\min \left(s_{\mathcal{A}}, s_{\mathcal{B}}\right)\right\} \cup\left\{0, \ldots, K_{\max }+1-\min \left(s_{\mathcal{A}}, s_{\mathcal{B}}\right)\right\}\right\}$

Thus, Times $\left(s_{\mathcal{A}}, s_{B}\right)$ consists of the integers in $\left\{0, \ldots, K_{\max }+1-\min \left(s_{\mathcal{A}}, s_{\mathcal{B}}\right)\right\}$ plus the distances between the points $p$ and the integers $1, \ldots, K_{\max }-\min \left(s_{\mathcal{A}}, s_{\mathcal{B}}\right)$, where $p$ is either a fractional part $\tau_{j}$ or the mid-point of some interval $\left[\tau_{i}, \tau_{i+1}\right]$ with $0 \leq i \leq n$. Intuitively, the distance $d$ between a mid-point $\frac{1}{2}\left(\tau_{i}+\tau_{i+1}\right)$ and an integer $h=1, \ldots, K_{\max }-\min \left(s_{\mathcal{A}}, s_{\mathcal{B}}\right)$ 
is used as a representative for all timestamps $t$ such that $h-\tau_{i+1}<t<h-\tau_{i}$ (formally, $\left(v_{\mathcal{A}} \| v_{\mathcal{B}}\right)+t \approx_{\mathcal{A} \| \mathcal{B}}\left(v_{\mathcal{A}} \| v_{\mathcal{B}}\right)+d$, where $v_{\mathcal{A}}$ and $v_{\mathcal{B}}$ are the clock valuations of $s_{\mathcal{A}}$ and $\left.s_{\mathcal{B}}\right)$.

Checking if $\mathcal{A} \preceq_{\sigma} \mathcal{B}$ for $\sigma \in\{0,1\}$ : let $H_{\sigma}^{\max }$ be the maximal timed alternating simulation for player $\sigma$ from $\mathcal{A}$ to $\mathcal{B}$. We show that $H_{\sigma}^{\max }$ is a computable union of regions.

DEFINITION 7.[Goodness] Let $\Gamma \subseteq \operatorname{Reg}_{\mathcal{A} \| \mathcal{B}}$ be a set of regions and let $R \in \Gamma$. We say that $R$ is good in $\Gamma$ w.r.t. player $\sigma \in\{0,1\}$ iff there is $\left(s_{\mathcal{A}}, s_{\mathcal{B}}\right) \in R$ such that:

1. for every move $m_{\sigma}^{\mathcal{A}}=\left(t, a, \delta_{\mathcal{A}}\right) \in \operatorname{Mov}_{\mathcal{A}}\left(\sigma, s_{\mathcal{A}}\right)$ with $t \in \operatorname{Times}\left(s_{\mathcal{A}}, s_{\mathcal{B}}\right)$, there is a matching move $m_{\sigma}^{\mathcal{B}}=\left(t, a, \delta_{\mathcal{B}}\right) \in \operatorname{Mov}_{\mathcal{B}}\left(\sigma, s_{\mathcal{B}}\right)$ such that for every move $m_{1-\sigma}^{\mathcal{B}}=\left(t^{\prime}, b, \delta_{\mathcal{B}}^{\prime}\right) \in$ $\operatorname{Mov}_{\mathcal{B}}\left(1-\sigma, s_{\mathcal{B}}\right)$ with $t^{\prime} \in \operatorname{Times}\left(s_{\mathcal{A}}, s_{\mathcal{B}}\right)$, there is a matching move $m_{1-\sigma}^{\mathcal{A}}=\left(t^{\prime}, b, \delta_{\mathcal{A}}^{\prime}\right) \in$ $\operatorname{Mov}_{\mathcal{A}}\left(1-\sigma, s_{\mathcal{A}}\right)$ so that for all $i=0,1$ with $m_{i}^{\mathcal{A}} \in J D M\left(m_{0}^{\mathcal{A}}, m_{1}^{\mathcal{A}}\right)$,

$\left(\operatorname{Next}_{\mathcal{A}}\left(s_{\mathcal{A}}, m_{i}^{\mathcal{A}}\right), \operatorname{Next}_{\mathcal{B}}\left(s_{\mathcal{B}}, m_{i}^{\mathcal{B}}\right)\right) \in R_{i}$ for some $R_{i} \in \Gamma$

Fix $\sigma \in\{0,1\}$ and let $\Omega_{\sigma}: 2^{\operatorname{Reg}_{\mathcal{A} \| \mathcal{B}}} \rightarrow 2^{\operatorname{Reg} \mathcal{g}_{\mathcal{A} \| \mathcal{B}}}$ be the monotone operator defined as follows: $\Omega_{\sigma}(\Gamma)=\{R \in \Gamma \mid R$ is good in $\Gamma$ w.r.t. player $\sigma\}$. We show that $\Omega_{\sigma}$ is computable and $H_{\sigma}^{\max }=\bigcup_{R \in \Gamma_{\max }} R$, where $\Gamma_{\max }$ is the greatest fixpoint of $\Omega_{\sigma}$. For this, we need the following crucial technical lemma, which extends the result given in [17] for timed simulation.

LEMMA 8. Let $\Gamma \subseteq \operatorname{Reg}_{\mathcal{A} \| \mathcal{B}}$ be a set of regions and $R \in \Gamma$ such that $R$ is good in $\Gamma$ w.r.t. player $\sigma$. Then, each $\left(s_{\mathcal{A}}, s_{\mathcal{B}}\right) \in R$ satisfies the condition obtained from Condition 1 in Definition 7 by removing the constraint that the timestamps have to be chosen in Times $\left(s_{\mathcal{A}}, s_{\mathcal{B}}\right)$.

Let $H \subseteq S_{\mathcal{A}} \times S_{\mathcal{B}}$ be a timed alternating simulation for player $\sigma$ from $\mathcal{A}$ to $\mathcal{B}$. We denote by $\Gamma_{H}$ the set $\Gamma_{H}=\left\{R \in \operatorname{Reg}_{\mathcal{A} \| \mathcal{B}} \mid R \cap H \neq \varnothing\right\}$. By Definition 7, the following holds.

LEMMA 9. If $H \subseteq S_{\mathcal{A}} \times S_{\mathcal{B}}$ is a timed alternating simulation for player $\sigma$ from $\mathcal{A}$ to $\mathcal{B}$, then $\Gamma_{H}$ is a fixpoint of $\Omega_{\sigma}$.

By Lemmata 8 and 9, we obtain the following results.

COROLlaRY 10. If $H \subseteq S_{\mathcal{A}} \times S_{\mathcal{B}}$ is a timed alternating simulation for player $\sigma$ from $\mathcal{A}$ to $\mathcal{B}$, then $\bigcup_{R \in \Gamma_{H}} R$ is a timed alternating simulation for player $\sigma$ from $\mathcal{A}$ to $\mathcal{B}$.

COROLlaRY 11. Let $\Gamma \subseteq \operatorname{Reg}_{\mathcal{A} \| \mathcal{B}}$ be a set of regions. Then, $\Omega_{\sigma}(\Gamma)=\Gamma$ iff $\bigcup_{R \in \Gamma} R$ is a timed alternating simulation for player $\sigma$ from $\mathcal{A}$ to $\mathcal{B}$.

By Corollary 10, $H_{\sigma}^{\max }$ is a union of regions in $\operatorname{Reg}_{\mathcal{A} \| \mathcal{B}}$, and by Corollary $11, H_{\sigma}^{\max }=$ $\bigcup_{R \in \Gamma_{\max }} R$, where $\Gamma_{\max }$ is the greatest fixpoint of $\Omega_{\sigma}$. Note that $\Gamma_{\max }$ can be obtained by iterative applications of $\Omega_{\sigma}$ starting with $\Gamma_{0}=\operatorname{Reg}_{\mathcal{A} \| \mathcal{B}}$. There can be at most $\left|\operatorname{Reg} g_{\mathcal{A} \| \mathcal{B}}\right|$ many iterations. Moreover, by Lemma 8, Condition 1 in Definition 7 is independent on what representative is chosen for the given equivalence class. Since $\left|\operatorname{Times}\left(s_{\mathcal{A}}, s_{\mathcal{B}}\right)\right|$ for $\left(s_{\mathcal{A}}, s_{\mathcal{B}}\right) \in$ $S_{\mathcal{A}} \times S_{\mathcal{B}}$ and $\left|\operatorname{Reg}_{\mathcal{A} \| \mathcal{B}}\right|$ are singly exponential in the sizes of $\mathcal{A}$ and $\mathcal{B}$, if follows that $\Omega_{\sigma}(\Gamma)$ for given $\Gamma \subseteq \operatorname{Reg}_{\mathcal{A} \| \mathcal{B}}$ can be computed in single exponential time in the sizes of $\mathcal{A}$ and $\mathcal{B}$. Since $\mathcal{A} \preceq_{\sigma} \mathcal{B}$ iff $\left(s_{0}^{\mathcal{A}}, s_{0}^{\mathcal{B}}\right) \in H_{\sigma}^{\text {max }}$, checking whether $\mathcal{A} \preceq_{\sigma} \mathcal{B}$ is in ExPTIME. We can show that the problem is also EXPTIME-hard by a straightforward and linear reduction from checking timed simulation between TT, which is ExPTIME-hard [14]. Thus, we obtain the following.

Theorem 12. Given two comparable TG $\mathcal{A}$ and $\mathcal{B}$ and player $\sigma \in\{0,1\}$, the problem of checking whether $\mathcal{A} \preceq_{\sigma} \mathcal{B}$ is ExPTIME-complete. 


\subsection{Logical characterization of timed alternating simulation}

In this subsection, we give a logical characterization of timed alternating simulation for a given player $\sigma \in\{0,1\}$ in terms of the fragment $\sigma$-TAMTL ${ }_{P}^{*}$ of TAMTL*.

TheOrem 13. Let $\mathcal{A}$ and $\mathcal{B}$ be two TG over $\left(\right.$ Act $\left._{0}, A c t_{1}\right)$ and $\sigma \in\{0,1\}$. Then, $\mathcal{A} \preceq_{\sigma} \mathcal{B}$ if and only if for every $\sigma$-TAMTL $L_{P}^{*}$ formula $\varphi, \mathcal{A} \models \varphi$ implies $\mathcal{B} \models \varphi$. Hence, $\mathcal{A} \preceq_{\sigma} \mathcal{B}$ if and only if for every $\sigma$-TAMTL ${ }_{P}^{*}$ formula $\widetilde{\varphi}, \mathcal{B} \models \widetilde{\varphi}$ implies $\mathcal{A} \models \widetilde{\varphi}$.

Sketched proof. For the direct implication $(\Rightarrow)$, it suffices to show that if $H$ is a timed alternating simulation for player $\sigma$ from $\mathcal{A}$ to $\mathcal{B}$, then the following holds:

1. for all $\sigma$-TAMTL ${ }_{P}^{*}$ (state) formulas $\varphi$ and $\left(s_{\mathcal{A}}, s_{\mathcal{B}}\right) \in H,\left(\mathcal{A}, s_{\mathcal{A}}\right) \models \varphi$ implies $\left(\mathcal{B}, s_{\mathcal{B}}\right) \models \varphi$.

2. for all path formulas $\psi$ of $\sigma$-TAMTL ${ }_{P}^{*}$ and for all infinite runs $\pi_{\mathcal{A}}$ of $\mathcal{A}$ and $\pi_{\mathcal{B}}$ of $\mathcal{B}$ s.t. $H\left(\pi_{\mathcal{A}}, \pi_{\mathcal{B}}\right)$ and $\operatorname{trace}\left(\pi_{\mathcal{A}}\right)=\operatorname{trace}\left(\pi_{\mathcal{B}}\right),\left(\mathcal{A}, \pi_{\mathcal{A}}\right) \models \psi$ implies $\left(\mathcal{B}, \pi_{\mathcal{B}}\right) \models \psi$.

The proof is by induction on the structure of formulas. The non-trivial case is that of state formulas of the form $\langle\langle\sigma\rangle\rangle \psi$ (recall that $\langle\langle\sigma\rangle\rangle_{r e}$ is a derivate operator in $\sigma$-TAMTL ${ }_{P}^{*}$ ). Assume that $\left(s_{\mathcal{A}}, s_{\mathcal{B}}\right) \in H$ and $\left(\mathcal{A}, s_{\mathcal{A}}\right) \models\langle\langle\sigma\rangle\rangle \psi$. Thus, there is a strategy $f_{\mathcal{A}}$ of player $\sigma$ in $\mathcal{A}$ such that for each outcome $\pi_{\mathcal{A}}$ of $f_{\mathcal{A}}$ from $s_{\mathcal{A}},\left(\mathcal{A}, \pi_{\mathcal{A}}\right) \models \psi$. Since $\left(s_{\mathcal{A}}, s_{\mathcal{B}}\right) \in H$, by Lemma 5 , there is a strategy $f_{\mathcal{B}}$ of player $\sigma$ in $\mathcal{B}$ such that for each outcome $\pi_{\mathcal{B}}$ of $f_{\mathcal{B}}$ from $s_{\mathcal{B}}$, there is an outcome $\pi_{\mathcal{A}}$ of $f_{\mathcal{A}}$ from $s_{\mathcal{A}}$ so that $H\left(\pi_{\mathcal{A}}, \pi_{\mathcal{B}}\right)$ and trace $\left(\pi_{\mathcal{A}}\right)=\operatorname{trace}\left(\pi_{\mathcal{B}}\right)$. By ind. hyp., Property 2 holds for the path formula $\psi$. Hence, evidently, the result follows.

For the converse implication $(\Leftarrow)$ of the theorem, assume that $\mathcal{A} \swarrow_{\sigma} \mathcal{B}$. Let $\sigma=0$ (the other case is symmetric). We need to prove that for some 0 -TAMTL* formula $\varphi, \mathcal{A} \models \varphi$ and $\mathcal{B} \not \models \varphi$. Consider the turn-based 0 -simulation game $G_{0}$ between the antagonist and the protagonist on page 90. By the results of Subsection 3.1 we can assume that the timestamps chosen by the antagonist are in the finite $\operatorname{set} \operatorname{Times}\left(s_{\mathcal{A}}, s_{\mathcal{B}}\right)$, where $\left(s_{\mathcal{A}}, s_{\mathcal{B}}\right)$ is the main current position of the game. It follows that $G_{0}$ is finitely-branching. Since $\mathcal{A} \swarrow_{0} \mathcal{B}$, the antagonist has a winning strategy $f$ starting from $\left(s_{0}^{\mathcal{A}}, s_{0}^{\mathcal{B}}\right)$, where $s_{0}^{\mathcal{A}}$ (resp., $\left.s_{0}^{\mathcal{B}}\right)$ is the initial state of $\mathcal{A}$ $($ resp., $\mathcal{B})$ whose clock-values are rational. Hence, the strategy-tree $T_{f}$ of $f$ from $\left(s_{0}^{\mathcal{A}}, s_{0}^{\mathcal{B}}\right)$ is finite, and (by def. of Times) the timestamps of the moves along the edges of $T_{f}$ are rational. We claim that for each node $x_{p}$ of $T_{f}$ labeled by a main position $p=\left(s_{\mathcal{A}}, s_{\mathcal{B}}\right) \in S_{\mathcal{A}} \times S_{\mathcal{B}}$, there is a 0 -TAMTL ${ }_{P}^{*}$ formula $\varphi_{p}$ such that $\left(\mathcal{A}, s_{\mathcal{A}}\right) \models \varphi_{p}$ and $\left(\mathcal{B}, s_{\mathcal{B}}\right) \not \models \varphi_{p}$. Hence, the result follows. The proof is by induction on the height of the subtree of $T_{f}$ rooted at node $x_{p}$. By construction, $x_{p}$ has exactly one child, say $x_{p}^{\prime}$, and the edge from $x_{p}$ to $x_{p}^{\prime}$ corresponds to a move $m_{\mathcal{A}}^{0}=\left(t, a, \delta_{\mathcal{A}}\right)$ for player 0 in $\operatorname{Mov}_{\mathcal{A}}\left(0, s_{\mathcal{A}}\right)$ with $t \in \operatorname{Times}\left(s_{\mathcal{A}}, s_{\mathcal{B}}\right) \subseteq \mathbb{Q}_{\geq 0}$ chosen by the antagonist in Step 1 on page 90. Moreover, the edges from $x_{p}^{\prime}$ to its children $y_{1}, \ldots, y_{n}$, if any, correspond to all and only the matching moves $m_{\mathcal{B}}^{0}=\left(t, a, \delta_{\mathcal{B}}\right) \in \operatorname{Mov}_{\mathcal{B}}\left(0, s_{\mathcal{B}}\right)$ of $m_{\mathcal{A}}^{0}$ for player 0 in $\mathcal{B}$ from $s_{\mathcal{B}}$. If $n=0$ (base case), there is no such a matching move. In this case, the $0-\mathrm{TAMTL}_{P}^{*}$ formula $\varphi_{p}$ satisfying the claim is $\langle\langle 0\rangle\rangle\left(\bigvee_{b \in A c t_{1}^{\perp}} \bigvee_{\kappa \in\{0,1\}}\langle(a,=t),(b, \geq 0), \kappa\rangle\right)$.

Now, assume that $n \geq 1$. By construction, for each $1 \leq i \leq n, y_{i}$ has a unique child $y_{i}^{\prime}$ and the edge from $y_{i}$ to $y_{i}^{\prime}$ is associated with some move $\left(t^{\prime}, b, \delta_{\mathcal{B}}^{\prime}\right) \in \operatorname{Mov}_{\mathcal{B}}\left(1, s_{\mathcal{B}}\right)$ (depending on $i$ ) with $t^{\prime} \in \operatorname{Times}\left(s_{\mathcal{A}}, s_{\mathcal{B}}\right) \subseteq \mathbb{Q}_{\geq 0}$ chosen by the antagonist in Step 3 on page 90. Moreover, the edges from $y_{i}^{\prime}$ to its children $z_{i, 1}, \ldots, z_{i, m_{i}}$ represent all and only the possible matching moves $\left(t^{\prime}, b, \delta_{\mathcal{A}}^{\prime}\right) \in \operatorname{Mov}_{\mathcal{A}}\left(1, s_{\mathcal{A}}\right)$ (for player 1 in $\mathcal{A}$ from $s_{\mathcal{A}}$ ) of the move $\left(t^{\prime}, b, \delta_{\mathcal{B}}^{\prime}\right) \in \operatorname{Mov}_{\mathcal{B}}\left(1, s_{\mathcal{B}}\right)$. Assume that for each $1 \leq i \leq n, m_{i} \geq 1$, i.e. $y_{i}^{\prime}$ is not a leaf (the 
other case being simpler). By construction, for each $1 \leq l \leq m_{i}, z_{i, l}$ has a unique child $z_{i, l}^{\prime}$, which is labeled by a main position in $S_{\mathcal{A}} \times S_{\mathcal{B}}$, and the edge from $z_{i, l}$ to $z_{i, l}^{\prime}$ corresponds to a choice $\kappa=0,1$ of the antagonist in Step 5 on page 90. We distinguish two cases:

- $\exists 1 \leq i \leq n . \forall 1 \leq l \leq m_{i}$ : the edge from $z_{i, l}$ to $z_{i, l}^{\prime}$ is associated with the choice $\kappa=1$;

- $\forall 1 \leq i \leq n . \exists 1 \leq l \leq m_{i}$ : the edge from $z_{i, l}$ to $z_{i, l}^{\prime}$ is associated with the choice $\kappa=0$.

Here, we focus on the first case. Let $m_{\mathcal{B}}^{1}=\left(t^{\prime}, b, \delta_{\mathcal{B}}^{\prime}\right) \in \operatorname{Mov}_{\mathcal{B}}\left(1, s_{\mathcal{B}}\right)$ be the move associated with the edge from $y_{i}$ to $y_{i}^{\prime}$, where $t^{\prime} \in \mathbb{Q}_{\geq 0}$, and $w_{\mathcal{B}}=N \operatorname{Next}_{\mathcal{B}}\left(s_{\mathcal{B}}, m_{\mathcal{B}}^{1}\right)$. By construction, $t^{\prime} \leq t$ and the nodes $z_{i, 1}^{\prime}, \ldots, z_{i, m_{i}}^{\prime}$ are labeled by positions $\left(w_{\mathcal{A}}^{1}, w_{\mathcal{B}}\right), \ldots,\left(w_{\mathcal{A}}^{m_{i}}, w_{\mathcal{B}}\right)$, respectively, where $w_{\mathcal{A}}^{1}, \ldots, w_{\mathcal{A}}^{m_{i}}$ are the states of $\mathcal{A}$ obtained from $s_{\mathcal{A}}$ applying all and only the matching moves $\left(t^{\prime}, b, \delta_{\mathcal{A}}^{\prime}\right) \in \operatorname{Mov}_{\mathcal{A}}\left(1, s_{\mathcal{A}}\right)$ of $m_{\mathcal{B}}^{1}$. By ind. hyp. for each $1 \leq l \leq m_{i}$, there is a 0 -TAMTL* formula $\phi_{l}$ s.t. $\left(\mathcal{A}, w_{\mathcal{A}}^{l}\right) \models \phi_{l}$ and $\left(\mathcal{B}, w_{\mathcal{B}}\right) \not \models \phi_{l}$. Let $\varphi_{p}$ be the 0 -TAMTL ${ }_{P}^{*}$ formula given by

$$
\langle\langle 0\rangle\rangle\left\{\left(\bigvee_{c \in A c t_{1}^{\perp}} \bigvee_{\kappa \in\{0,1\}}\langle(a,=t),(c, \geq 0), \kappa\rangle\right) \wedge\left(\left\langle(a,=t),\left(b,=t^{\prime}\right), 1\right\rangle \rightarrow \bigcirc\left(\phi_{1} \vee \ldots \vee \phi_{m_{i}}\right)\right)\right\}
$$

Evidently, $\left(\mathcal{A}, s_{\mathcal{A}}\right) \models \varphi_{p}$. Moreover, $\left(\mathcal{B}, s_{\mathcal{B}}\right) \not \models \varphi_{p}$, since for every strategy of player 0 in $\mathcal{B}$ which initially selects from $s_{\mathcal{B}}$ a move of the form $(t, a, \delta)$, there is an outcome from $s_{\mathcal{B}}$ of the form $\pi=s_{\mathcal{B}},\left\langle(t, a, \delta),\left(t^{\prime}, b, \delta_{\mathcal{B}}\right), 1\right\rangle, w_{\mathcal{B}}, \ldots$, where by hypothesis $w_{\mathcal{B}} \not \models\left(\phi_{1} \vee \ldots \vee \phi_{m_{i}}\right)$.

From the proof of Theorem 13, it follows that timed alternating simulation for player $\sigma$ can also be logically characterized by the small fragment of $\sigma$-TAMTL ${ }_{P}^{*}$ which only uses the boolean connectives, the next temporal modality $\bigcirc$, and the strategy quantifier $\langle\langle\sigma\rangle\rangle$.

\section{Model checking TG against TAMTL}

Fix a TG $\mathcal{A}_{\text {in }}$ over $\left(A c t_{0}, A c t_{1}\right)$ and a TAMTL formula $\varphi$. By [3], w.l.o.g. we can assume that the constants occurring in $\varphi$ are natural numbers. Moreover, we can assume that $\mathcal{A}_{\text {in }}$ uses a clock $x_{\text {div }}$, which is reset whenever the constraint $x_{\text {div }} \geq 1$ holds. Let $\mathcal{A}$ be the TG obtained from $\mathcal{A}_{\text {in }}$ by simply adding a new clock, say $x_{\varphi}$ (note that $x_{\varphi}$ is never used by $\mathcal{A}$ ). Let $K_{\max }$ be the largest constant occurring in $\mathcal{A}$ and $\varphi$. We denote by $\operatorname{Reg}_{\mathcal{A}_{\text {in }}}$ (resp., $\operatorname{Reg}_{\mathcal{A}}$ ) the finite set of equivalences classes of the region equivalence on the set $S_{\mathcal{A}_{i n}}$ (resp., $S_{\mathcal{A}}$ ) of states of $\mathcal{A}_{\text {in }}$ (resp., $\mathcal{A}$ ) w.r.t. the constant $K_{\max }$ [3], which is defined similarly to the set $\operatorname{Reg}_{\mathcal{A} \| \mathcal{B}}$ in Subsection 3.1. We show that checking whether $\mathcal{A}_{\text {in }} \models \varphi$ (model-checking problem) can be reduced to solving finite-state games w.r.t. regular objectives. For this, we associate to $\mathcal{A}$ two finite-state games which abstract away from precise time information.

Let $R \in \operatorname{Reg}_{\mathcal{A}}$. A region $R^{\prime} \in \operatorname{Reg}_{\mathcal{A}}$ is an abstract time-successor of $R$, written $R \leq R^{\prime}$, if there is $(q, v) \in R$ such that for some $t \in \mathbb{R}_{\geq 0},(q, v+t) \in R^{\prime}$ and $v+t^{\prime} \models \operatorname{Inv}(q)$ for each $0<t^{\prime}<t$. By [3], the previous condition is independent on what representative is chosen in $R$. Moreover, if $R \leq R^{\prime}$ and $R \leq R^{\prime \prime}$, then either $R^{\prime} \leq R^{\prime \prime}$ or $R^{\prime \prime} \leq R^{\prime}$. The set of abstract moves available to player $\sigma$ in $R$, written $\operatorname{Mov}_{\mathcal{A}}^{a b s}(\sigma, R)$, is the set of triples $\left(R^{\prime}, a, \delta\right) \in$ $\operatorname{Reg}_{\mathcal{A}} \times A c t_{\sigma}^{\perp} \times \Delta$, such that $R \leq R^{\prime}, \delta=\left(q, a, g, Y, q^{\prime}\right), q$ is the location associated with $R^{\prime}$, and $g$ holds in $R^{\prime}$. Given $m=\left(R^{\prime}, a, \delta\right) \in \operatorname{Mov}_{\mathcal{A}}^{a b s}(\sigma, R)$ with $\delta=\left(q, a, g, Y, q^{\prime}\right)$, we denote by $\operatorname{Next} t_{\mathcal{A}}^{a b s}(R, m)$ the unique region $R^{\prime \prime}$ such that there is $(q, v) \in R^{\prime}$ so that $(q, v[Y:=0]) \in R^{\prime \prime}$. By [3], the previous condition is independent on what representative is chosen in $R^{\prime}$.

Let $\sigma \in\{0,1\}$. The finite-state turn-based two-player game $\mathcal{A}_{\sigma}^{a b s}=\left\langle P_{\sigma}=P_{\sigma}^{\sigma} \cup P_{\sigma}^{1-\sigma}, E_{\sigma}\right\rangle$ is defined as follows: $P_{\sigma}^{\sigma}=\operatorname{Reg}_{\mathcal{A}} \times\{0,1\}$ is the set of states (or positions) for player $\sigma$, 
$P_{\sigma}^{1-\sigma}=\left\{\langle R, m, l\rangle \mid R \in \operatorname{Reg}_{\mathcal{A}}, m \in \operatorname{Mov}_{\mathcal{A}}^{a b s}(\sigma, R)\right.$, and $\left.l \in\{0,1\}\right\}$ is the set of positions for player $1-\sigma$, and $E_{\sigma} \subseteq\left(P_{\sigma}^{\sigma} \times P_{\sigma}^{1-\sigma}\right) \cup\left(P_{\sigma}^{1-\sigma} \times P_{\sigma}^{\sigma}\right)$ consists of following edges:

- $(R, l) \rightarrow(R, m, l)$ for all $R \in \operatorname{Reg}_{\mathcal{A}}, m \in \operatorname{Mov}_{\mathcal{A}}^{a b s}(\sigma, R)$, and $l \in\{0,1\}$;

- $\left(R,\left\langle R_{1}, a, \delta_{1}\right\rangle, l\right) \rightarrow\left(R^{\prime}, l^{\prime}\right)$ iff $\exists\left\langle R_{2}, b, \delta_{2}\right\rangle \in \operatorname{Mov}_{\mathcal{A}}^{a b s}(1-\sigma, R)$ s.t. either $l^{\prime}=\sigma, R_{1} \leq R_{2}$, and $R^{\prime}=\operatorname{Next}_{\mathcal{A}}^{a b s}\left(R,\left\langle R_{1}, a, \delta_{1}\right\rangle\right)$, or $l^{\prime}=1-\sigma, R_{2} \leq R_{1}$, and $R^{\prime}=\operatorname{Next}_{\mathcal{A}}^{a b s}\left(R,\left\langle R_{2}, b, \delta_{2}\right\rangle\right)$. A strategy for player $\sigma$ in $\mathcal{A}_{\sigma}^{a b s}$ is a function $f: P_{\sigma}^{*} \cdot P_{\sigma}^{\sigma} \rightarrow P_{\sigma}^{1-\sigma}$ such that for each $\pi=\pi^{\prime} \cdot p \in$ $P_{\sigma}^{*} \cdot P_{\sigma}^{\sigma}, p \rightarrow f(\pi)$ is an edge of $\mathcal{A}_{\sigma}^{a b s}$. For each $p \in P_{\sigma}$, the set Outcomes ${ }_{\mathcal{A}_{\sigma}^{a b s}}(\sigma, p, f)$ of infinite outcomes of $f$ from $p$ is defined in the usual way. For a finite set of propositions Prop, a labeling function $L: P_{\sigma} \rightarrow 2^{\text {Prop }}$, a standard LTL formula $\xi$ over Prop , and position $p \in P_{\sigma}$, we say that the strategy $f$ is winning in $p$ w.r.t. $L$ and the objective $\xi$ if for each outcome $p_{0}, p_{1}, \ldots \in$ Outcomes $\mathcal{A}_{\sigma}^{a b s}(\sigma, p, f), L\left(p_{0}\right), L\left(p_{1}\right), \ldots$ satisfies $\xi$. The following two lemmata show the connection between the strategies of player $\sigma$ in $\mathcal{A}$ and the strategies of player $\sigma$ in $\mathcal{A}_{\sigma}^{a b s}$.

LEMmA 14. Let $\sigma \in\{0,1\}, f$ be a strategy of player $\sigma$ in $\mathcal{A}, R_{0} \in R e g_{\mathcal{A}^{\prime}}$ and $s_{0} \in R_{0}$. Then, there is a strategy $f_{a b s}$ of player $\sigma$ in $\mathcal{A}_{\sigma}^{a b s}$ such that for each path $\pi_{a b s}=\left(R_{0}, 0\right), p_{0},\left(R_{1}, \underline{\sigma_{1}}\right), p_{1}$, $\left(R_{2}, \underline{\sigma_{2}}\right) \ldots \in$ Outcomes $_{\mathcal{A}_{\sigma}^{a b s}}\left(\sigma,\left(R_{0}, 0\right), f_{a b s}\right)$, there exists a run $\pi \in$ Outcomes $_{\mathcal{A}}\left(\sigma, s_{0}, f\right)$ of the form $\pi=s_{0},\left\langle m_{1}^{0}, m_{1}^{1}, \underline{\sigma_{1}}\right\rangle, s_{1},\left\langle m_{2}^{0}, m_{2}^{1}, \underline{\sigma_{2}}\right\rangle, \ldots$ so that for each $h \geq 1, s_{h} \in R_{h}$.

LEMMA 15. Let $\sigma \in\{0,1\}$ and $f_{a b s}$ be a strategy of player $\sigma$ in $\mathcal{A}_{\sigma}^{a b s}$, and $R_{0} \in \operatorname{Reg}_{\mathcal{A}}$. Then, there is a strategy $f$ of player $\sigma$ in $\mathcal{A}$ s.t. for each $\pi=s_{0},\left\langle m_{1}^{0}, m_{1}^{1}, \underline{\sigma_{1}}\right\rangle, s_{1},\left\langle m_{2}^{0}, m_{2}^{1}, \underline{\sigma_{2}}\right\rangle, \ldots \in$ Outcomes $_{\mathcal{A}}\left(\sigma, s_{0}, f\right)$ with $s_{0} \in R_{0}$, there is $\pi_{a b s} \in$ Outcomes $\left._{\mathcal{A}_{\sigma}^{a b s}}\left(\sigma, \overline{(R}_{0}, 0\right), f_{a b s}\right)$ of the form $\pi_{a b s}=\left(R_{0}, 0\right), p_{0},\left(R_{1}, \underline{\sigma_{1}}\right), p_{1},\left(R_{2}, \underline{\sigma_{2}}\right) \ldots$ so that for each $h \geq 1, s_{h} \in R_{h}$.

THEOREM 16. The set of states $s_{\text {in }}$ of $\mathcal{A}_{\text {in }}$ such that $\left(\mathcal{A}_{\text {in }}, s_{\text {in }}\right) \models \varphi$ is a union of regions in

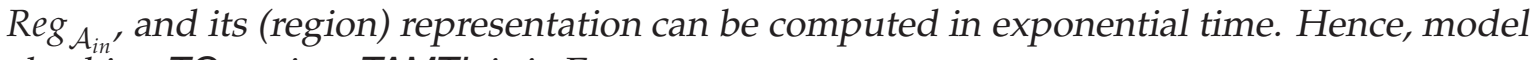
checking TG against TAMTL is in EXPTIME.

PROOF. We prove by induction on the structure of the formulas that the result holds for each state subformula $\phi$ of $\varphi$. Here, we illustrate the case in which $\phi=\langle\langle\sigma\rangle\rangle_{\text {re }}\left(\phi_{1} \mathcal{U}_{I} \phi_{2}\right)$ for some $\sigma \in\{0,1\}$. For $s \in S_{\mathcal{A}}$, we denote by $\operatorname{Proj}(s)$ the associated state in $S_{\mathcal{A}_{\text {in }}}$. Let $S_{\mathcal{A}}\left[x_{\varphi}:=\right.$ $0]$ be the set of states in $S_{\mathcal{A}}$ such that the value of clock $x_{\varphi}$ is 0 . Note that for each $s \in S_{\mathcal{A}}$, $(\mathcal{A}, s) \models \phi$ iff $\left(\mathcal{A}_{\text {in }}, \operatorname{Proj}(s)\right) \models \phi$. By ind. hyp. it follows that for each $i=1,2$, the set of states $s \in S_{\mathcal{A}}$ such that $(\mathcal{A}, s)=\phi_{i}$ is a union of regions in $\operatorname{Reg}_{\mathcal{A}}$ whose representation can be computed in exponential time. Evidently, it suffices to show that the last condition continues to hold for the set of states $s$ in $S_{\mathcal{A}}\left[x_{\varphi}:=0\right]$ such that $(\mathcal{A}, s) \models\langle\langle\sigma\rangle\rangle_{r e}\left(\phi_{1} \mathcal{U}_{I} \phi_{2}\right)$. Note that by the previous observations, for each $s_{0} \in S_{\mathcal{A}}\left[x_{\varphi}:=0\right],\left(\mathcal{A}, s_{0}\right) \models\langle\langle\sigma\rangle\rangle_{r e}\left(\phi_{1} \mathcal{U}_{\text {I }} \phi_{2}\right)$ iff there is a strategy $f$ of player $\sigma$ in $\mathcal{A}$ such that for each $\pi=s_{0},\left\langle m_{1}^{0}, m_{1}^{1}, \sigma_{1}\right\rangle, s_{1},\left\langle m_{2}^{0}, m_{2}^{1}, \sigma_{2}\right\rangle, \ldots \in$ $\operatorname{Outcomes}_{\mathcal{A}}\left(\sigma, s_{0}, f\right)$, the associate sequence $\operatorname{Reg}\left(s_{0}\right), \sigma_{1}, \operatorname{Reg}\left(s_{1}\right), \sigma_{2}, \ldots$, where $\operatorname{Reg}\left(s_{j}\right)$ is the region of $s_{j}$, satisfies the following: either (1) for infinitely many $j \geq 0, \operatorname{Reg}\left(s_{j}\right)$ satisfies the constraint $x_{\text {div }} \geq 1$, and there is $k>0$ such that $\operatorname{Reg}\left(s_{k}\right)$ satisfies $\phi_{2}$ and the constraint $x_{\varphi} \in I$, and $\operatorname{Reg}\left(s_{h}\right)$ satisfies $\phi_{1}$ for each $0<h<k$, or (2) there is $k \geq 0$ such that for each $j \geq k$, $\sigma_{j} \neq \sigma$ and $\operatorname{Reg}\left(s_{j}\right)$ satisfies $x_{\text {div }}<1$. Let $L: P_{\sigma} \rightarrow\left\{p_{\phi_{2}}, p_{\phi_{1}},\left(x_{\text {div }} \geq 1\right),\left(x_{\varphi} \in I\right), 0,1\right\}$ be the labeling of $\mathcal{A}_{\sigma}^{a b s}$ defined in the obvious way. Then, by Lemmata 14 and 15, for all regions $R_{0} \in \operatorname{Reg}_{\mathcal{A}}$ satisfying $x_{\varphi}=0$ and $s_{0} \in R_{0}$, it holds that $\left(\mathcal{A}, s_{0}\right) \models\langle\langle\sigma\rangle\rangle_{r e}\left(\phi_{1} \mathcal{U}_{I} \phi_{2}\right)$ iff there is a winning strategy $f_{a b s}$ of player $\sigma$ in $\mathcal{A}_{\sigma}^{a b s}$ in position $\left(R_{0}, 0\right)$ w.r.t. the labeling $L$ and the LTL objective: $\left[\square \diamond\left(x_{\text {div }} \geq 1\right) \wedge\left(p_{\phi_{1}} \mathcal{U}\left(p_{\phi_{2}} \wedge\left(x_{\varphi} \in I\right)\right)\right)\right] \vee\left[\diamond \square\left(\neg\left(x_{\text {div }} \geq 1\right) \wedge(1-\sigma)\right)\right]$ 
Since LTL finite-state games for a fixed LTL formula can be solved in polynomial time [18] and since the size of $\mathcal{A}_{\sigma}^{a b s}$ is exponential in the size of $\mathcal{A}_{\text {in }}$, the result follows.

\section{References}

[1] L. Aceto and A. Jeffrey. A Complete Axiomatization of Timed Bisimulation for a Class of Timed Regular Behaviours. Theoretical Computer Science, 152(2):251-268, 1995.

[2] R. Alur, C. Courcoubetis, and D.L. Dill. Model-checking in dense real-time. Information and Computation, 104(1):2-34, 1993.

[3] R. Alur and D. Dill. A theory of timed automata. Theoretical Computer Science, 126(2):183-235, 1994.

[4] R. Alur, T.A. Henzinger, and O. Kupferman. Alternating-time temporal logic. Journal of the ACM, 49(5):672-713, 2002.

[5] R. Alur, T.A. Henzinger, O. Kupferman, and M .Y. Vardi. Alternating Refinement Relations. In Proc. 9th CONCUR, LNCS 1466, pp. 163-178, 1998.

[6] P. Bulychev, T. Chatain, A. David, and K.G. Larsen. Checking simulation relation between timed game automata. In Proc. 7th FORMATS, LNCS, 2009. To appear

[7] K. Čerāns. Decidability of Bisimulation Equivalences for Parallel Timer Processes. In Proc. 4th CAV, LNCS 663, pp. 302-315, 1992.

[8] L. de Alfaro, L. Dias da Silva, M. Faella, A. Legay, P. Roy, and M. Sorea. Sociable Interfaces. In Proc. 5th FROCOS, LNCS 3717, pp. 81-105, 2005.

[9] L. de Alfaro, M. Faella, T.A. Henzinger, R. Majumdar, and M. Stoelinga. The Element of Surprise in Timed Games. In Proc. 14th CONCUR, LNCS 2761, pp. 142-156, 2003.

[10] L. de Alfaro and T. A. Henzinger. Interface automata. In Proc. 9th FSE, ACM , pp. 109120, 2001.

[11] E.A. Emerson and J.Y. Halpern. Sometimes and Not Never Revisited: On Branching Versus Linear Time. Journal of the ACM, 33(1):151-178, 1986.

[12] T.A. Henzinger and V.S. Prabhu. Timed Alternating-Time Temporal Logic. In Proc. 4th FORMATS, LNCS 4202, pp. 1-17, 2006.

[13] R. Koymans. Specifying real-time properties with metric temporal logic. Real-Time Systems, 2(4):255-299, 1990.

[14] F. Laroussinie and Ph. Schnoebelen. The State Explosion Problem from Trace to Bisimulation Equivalence. In Proc. 3rd FOSSACS, LNCS 1784, pp. 192-207, 2000.

[15] J. Ouaknine and J. Worrell. On Metric Temporal Logic and Faulty Turing Machines. In Proc. 9th FOSSACS, LNCS 3921, pp. 217-230, 2006.

[16] J. Ouaknine and J. Worrell. On the Decidability of Metric Temporal Logic. In Proc. 20th LICS, pp. 188-197, IEEE Computer Society Press, 2005.

[17] S. Tasiran, R. Alur, R .P. Kurshan, and R.K. Brayton. Verifying Abstractions of Timed Systems. In Proc. 7th CONCUR, LNCS 1119, pp. 546-562,1996.

[18] W. Thomas. Automata on Infinite Objects. Handbook of Theoretical Computer Science: Formal Models and Sematics, B:133-192, 1990. 